\title{
Blood Pressure and Left Ventricular Characteristics in Young Patients with Classical Congenital Adrenal Hyperplasia due to 21-Hydroxylase Deficiency
}

\author{
Graziamaria Ubertini, ${ }^{1}$ Carla Bizzarri, ${ }^{1}$ Armando Grossi, ${ }^{1}$ Fabrizio Gimigliano, ${ }^{2}$ \\ Lucilla Ravà, ${ }^{3}$ Danilo Fintini, ${ }^{4}$ and Marco Cappa ${ }^{1}$ \\ ${ }^{1}$ Department of Pediatrics, Unit of Endocrinology and Diabetes, Bambino Gesù Children's Hospital, School of Medicine, \\ University of Rome Tor Vergata, P.zza S. Onofrio 4, 00165 Rome, Italy \\ ${ }^{2}$ Cardiology Unit, Bambino Gesù Children's Hospital, Rome, Italy \\ ${ }^{3}$ Epidemiology Unit, Bambino Gesù Children's Hospital, Rome, Italy \\ ${ }^{4}$ Sport Medicine Unit, Bambino Gesù Children's Hospital, Rome, Italy \\ Correspondence should be addressed to Carla Bizzarri, bizzarri@opbg.net
}

Received 19 August 2009; Accepted 11 December 2009

Recommended by Peter Allen Lee

\begin{abstract}
High steroid doses are often necessary in congenital adrenal hyperplasia (CAH) to suppress androgens and may increase blood pressure (BP). We evaluated 24-hour BP profile (ambBP), BP during exercise (excBP), and echocardiography in 20 young CAH patients. Systolic and diastolic BP during ambBP and excBP was normal in all patients. None presented myocardial hypertrophy. Nocturnal diastolic BP was affected by testosterone ( $P: .016,95 \%$ CI: 0.002 to $0.021, \beta=0.01)$. Left ventricular mass (LVM ) was affected by height SDS ( $P: .007,95 \%$ CI: 2.67 to $14.17, \beta=8.42)$, age $(P:<.0001,95 \%$ CI: 2.12 to $5.82, \beta=3.97)$, and testosterone $(P: .008,95 \%$ CI: 0.01 to $0.09, \beta=0.053)$. Left ventricular mass index (LVMI) correlated with BMI SDS ( $P: .044,95 \%$ CI: 0.09 to $6.17, \beta=3.13)$ and testosterone ( $P: .031,95 \%$ CI: 0.002 to $0.035, \beta=0.018)$. Hydrocortisone dose did not influence ambBP, excBP, or myocardial hypertrophy.
\end{abstract}

Copyright ( $) 2009$ Graziamaria Ubertini et al. This is an open access article distributed under the Creative Commons Attribution License, which permits unrestricted use, distribution, and reproduction in any medium, provided the original work is properly cited.

\section{Introduction}

The 21 hydroxylase deficiency is the most common enzyme defect causing congenital adrenal hyperplasia (CAH). It is distinguished by the severity of the enzyme defect, in classical and non classical form. Approximately $70 \%$ individuals with classical CAH are affected by the more severe salt-wasting (SW) form, characterized by androgen hyper secretion and impaired synthesis of both glucocorticoids (GCs) and mineralcorticoids (MCs). About 20\%-30\% patients are affected by the milder simple virilizing (SV) form, where the excessive androgen production results in virilization, but the synthesis of MCs is sufficient to avoid salt wasting crises. The non classical form is characterized by later and milder signs of androgen excess as precocious pubarche [1].
The treatment of CAH is based on GCs therapy, in order to suppress the androgen production trough the inhibition of CRH and consequently ACTH. The therapy must be balanced to avoid poor linear growth, obesity and hypertension.

Several factors may interfere with the physiological control of $\mathrm{BP}$ in patients affected by $\mathrm{CAH}$ due to 21 hydroxilase deficiency: (a) over treatment with GCs and MCs; (b) elevated GCs levels that affect the MCs receptor (MR); (c) impaired adrenomedullary function, with a negative impact on sympathetic control of BP; (d) obesity itself [2-5].

An alteration in endothelial function has been demonstrated in GCs excess. Dexamethasone induces overproduction of reactive oxygen species causing dysregulation of the endothelial function. A reduction of nitric oxide levels, with a reduction of arteriolar vasodilatation has been demonstrated in GCs-induced hypertension [6]. 
MR binds progesterone, deoxycorticosterone (DOC), corticosterone, cortisol and aldosterone with a similar high affinity, but only DOC and aldosterone are full agonists of the MR. Corticosterone and cortisol have lower transcriptional activity on the MR, and elevated levels of these steroids are necessary to affect the MR. Hypertension in Cushing syndrome is mainly due to the effects of the elevated GCs levels on MR $[2,7]$. High serum leptin and insulin levels and low plasma epinephrine levels have been demonstrated in $\mathrm{CAH}$. They are probably due to the chronic adrenomedullary hypofunction, associated with the impaired synthesis of cortisol that normally induces the formation of adrenal medulla [8]. These alterations could contribute to determine insulin resistance, metabolic syndrome and cardiovascular disease.

Little is known about early BP and cardiovascular changes in $\mathrm{CAH}$ patients and their correlation with androgen levels and chronic steroid therapy.

\section{Aim of the Study}

Aim of our study was to search for early cardiovascular changes in young CAH patients, by the analysis of ambulatory 24-hour BP profile (ambBP), BP levels during physical exercise (excBP), and echocardiography. We investigated if androgen levels and GCs therapy could affect BP and heart function parameters.

\section{Patients}

We analysed 20 patients (14 females and 6 males), affected by classical CAH due to 21 hydroxylase deficiency (15 SW, 5 SV). All participants were Caucasian of Italian ancestry. The diagnosis was confirmed by molecular analysis in all patients. Seven females and 2 males were pubertal. They all received hydrocortisone (HC) therapy, twice or thrice daily at a mean dosage of $14.16 \pm 5.46 \mathrm{mg} / \mathrm{m}^{2} /$ day. The 15 patients with SW CAH assumed also fludrocortisone at a mean dose of $0.06 \pm 0.02 \mathrm{mg} /$ day. The institutional review board approved the study, informed assent and consent were obtained from the subjects and their parents or guardians. Anthropometric, clinical and hormonal characteristics of our patients are summarized in Table 1.

\section{Hormone Assay}

Commercial kits were used for the estimation of $17-\mathrm{OH}-$ progesterone (RIA, ICN-Pharmaceutical Inc.) and plasma renin (RIA, Sanofi-Pasteur). Mean intra- and interassay coefficients of variation where $8.8 \%$ and $12 \%$ for $17-\mathrm{OH}-$ progesterone, $7,9 \%$ and $10 \%$ for plasma renin. Testosterone levels were measured by chemiluminescence immunoassay (Centaur-Bayer), intra and interassay coefficients of variation were $3.8 \%$ and $5 \%$, respectively. ACTH concentrations were determined by immonoradiometric assay (ACTH IRMA, Nichols Institute Diagnostics), with a sensitivity of $1 \mathrm{pg} / \mathrm{mL}$. Intra- and interassay variability $3.2 \%$ and $6.8 \%$, respectively.
TABLE 1: Anthropometric clinical and hormonal characteristics of the study group.

\begin{tabular}{lcc}
\hline & Mean $( \pm \mathrm{SD})$ & Median $($ range $)$ \\
\hline Age (years) & $13.38( \pm 4.11)$ & $13.62(5.1-17.49)$ \\
Height SDS & $-0.27( \pm 1.23)$ & $-0.73(-1.63+2.24)$ \\
BMI SDS & $1.05( \pm 0.82)$ & $1.02(-0.14+2.46)$ \\
17-OH-progesterone & $16.71( \pm 16.78)$ & $11(0.2-54)$ \\
$(\mathrm{ng} / \mathrm{mL})$ & & \\
ACTH $(\mathrm{pg} / \mathrm{mL})$ & $88.09( \pm 50.98)$ & $64.0(1.6-310)$ \\
Plasma renin $(\mathrm{pg} / \mathrm{mL})$ & $53.43( \pm 43.52)$ & $22.5(1.3-450)$ \\
Testosterone $(\mathrm{ng} / \mathrm{dL})$ & $85.71( \pm 65.19)$ & $73.06(10-225.3)$ \\
Hydrocortisone dose & $14.16( \pm 5.46)$ & $13.58(10.06-21.3)$ \\
$\left(\mathrm{mg} / \mathrm{m}^{2} /\right.$ day $)$ & & \\
\hline
\end{tabular}

\section{Definition of Hypertension in Children}

The diagnosis of hypertension in paediatric age has to take into consideration the criteria to define normal BP levels in children. The first age-related norms for BP in children were developed in 1977 by the Task Force on Blood pressure Control in Children [9].

The standards were revised in 1987 by a second task force that evaluated data from 70000 White, African American and Mexican-American children [9]. In this revision, tables of normal BP according to age and gender were published. High BP was defined as systolic and/or diastolic BP between the 90th and 95th centile for age and gender. Hypertension was defined as systolic and/or diastolic BP greater than the 95th centile for age and gender. In the last revision, published in 1996, height was added to age and gender as a category in the standard BP tables [9].

Finally, to analyse BP in childhood, we have now to take in account of gender, age and height. Obesity is considered as an independent additional risk factor for hypertension in children, but weight or BMI are not included as categories in the tables of normal BP [10].

\section{Methods}

All CAH patients underwent ambBP profile during their normal daily activities, for the analysis of mean diurnal and nocturnal systolic and diastolic BP (SBP and DBP).

They also underwent endurance treadmill exercise by Bruce test (excBP), for the evaluation of BP levels at maximal heart rate. Bruce test consists in a progressive multistage treadmill test to volitional exhaustion [11]. Seven 3-minutes stages were used, in which the speed and inclination were increased in a stepwise fashion as follows: stage 1 $(2.7 \mathrm{~km} / \mathrm{h}$ and $10 \%$ inclination $)$; stage $2(4.0 \mathrm{~km} / \mathrm{h}$ and $12 \%$ inclination); stage $3(5.4 \mathrm{~km} / \mathrm{h}$ and $14 \%$ inclination); stage $4(6.7 \mathrm{~km} / \mathrm{h}$ and $16 \%$ inclination $)$; stage $5(8.0 \mathrm{~km} / \mathrm{h}$ and $18 \%$ inclination); stage $6(8.8 \mathrm{~km} / \mathrm{h}$ and $20 \%$ inclination); stage $7(10.6 \mathrm{~km} / \mathrm{h}$ and $22 \%$ inclination). Heart rate was continuously measured throughout the test, using a cardio tester (Polar, Electro Oy, Finland). The treadmill was stopped when each participant refused to continue the exercise 
test, despite verbal encouragement from the research staff. Maximal heart rate (beats/min), SBP and DBP were recorded at the end of the test. All tests were closely physicianmonitored and performed in the morning, after an overnight fast. The morning doses of $\mathrm{HC}$ and fluodrocortisone were administered at the end of the test. All patients familiarized with treadmill at least one week before the test, in order to avoid psychological stress. BP values were compared to Task Force references for after endurance exercise BP [10].

All participants were studied by echocardiography using a Philips iE33 Echocardiography System, in order to evaluate heart function and myocardial hypertrophy. Two$\mathrm{D}$ guided M-mode measurements of the left ventricular internal dimension were made during diastole, according to established methods by the American Society of Echocardiography [12]. Left ventricular mass (LVM) increases during growth and thus normal must be defined in the context of body size. LVM has been adjusted for height, body surface area and BMI raised to various powers. Height ${ }^{2.7}$ (in meters) has been validated as an indicator of lean body mass and has been recommended for indexing LVM. Use of height ${ }^{2.7}$ to index LVM also minimizes the effect of age, gender and race [13]. We determined the following measurements on $\mathrm{M}$ mode tracing:

(a) interventricular septum thickness (IVS), in millimeters;

(b) left ventricular posterior wall thickness (LVPW), in millimeters;

(c) left ventricular mass (LVM): calculated from measurement of the left ventricle (LV) by using the truncated ellipsoid methods by two-dimensional (2D) echocardiography recommended from the American Society of Echocardiography [12], in grams;

(d) left ventricular mass index (LVMI): representing LVM corrected for height (= LVM/meters ${ }^{2.7}$ ); left ventricular hypertrophy was defined as LVMI $>95^{\circ}$ centile for paediatric age [14], that is $>38.6 \mathrm{~g} / \mathrm{m}^{2.7}$.

\section{Statistical Analyses}

Statistical analyses were conducted using STATA Statistical Software Package, Release 10.1 (STATA, College station, TX, USA). Clinical and hormonal data are presented both as means \pm standard deviation (SD) and medians (range), although we confirmed by Skewness/Kurtosis tests that all the analyzed variables were normally distributed. $T$-test was used to analyze the gender related differences. Multivariable linear regression models were fitted in order to evaluate the independent effect of each covariate (age, height SDS, BMI SDS, testosterone, 17-OH-progesterone, ACTH, HC dose) on ambBP profile, excBP and myocardial hypertrophy parameters (IVS, LVPW, LVM, LVMI). A $P$ value $<.05$ was considered significant.
TABLE 2: Results of the 24-hours ambBP profile, excBP and echocardiography in $\mathrm{CAH}$ patients.

\begin{tabular}{lcc}
\hline & Mean \pm SD & Median (range) \\
\hline $\begin{array}{l}\text { Mean diurnal SBP } \\
\text { (mmHg) }\end{array}$ & $107.29 \pm 7.14$ & $109(95-122)$ \\
$\begin{array}{l}\text { Mean diurnal DBP } \\
\text { (mmHg) }\end{array}$ & $62.57 \pm 5.08$ & $61(56-72)$ \\
$\begin{array}{l}\text { Mean nocturnal SBP } \\
\text { (mmHg) }\end{array}$ & $97.43 \pm 7.43$ & $99.0(85-116)$ \\
$\begin{array}{l}\text { Mean nocturnal DBP } \\
\text { (mmHg) }\end{array}$ & $53.33 \pm 4.44$ & $52(45-64)$ \\
$\begin{array}{l}\text { Duration of exercise } \\
\text { (minutes) }\end{array}$ & $10.02 \pm 3.62$ & $10(75-14)$ \\
$\begin{array}{l}\text { Maximal heart rate } \\
\text { (beats/min) }\end{array}$ & $187.19 \pm 7.05$ & $188(170-200)$ \\
$\begin{array}{l}\text { SBP at maximal heart rate } \\
\text { (mmHg) }\end{array}$ & $132.38 \pm 18.14$ & $130(100-170)$ \\
$\begin{array}{l}\text { DBP at maximal heart rate } \\
\text { (mmHg) }\end{array}$ & $76.38 \pm 10.89$ & $80(60-100)$ \\
$\begin{array}{l}\text { LVM (g) } \\
\left.\text { LVMI (g/m }{ }^{2.7}\right)\end{array}$ & $76.22 \pm 24.60$ & $75.9(35-119)$ \\
$\begin{array}{l}\text { LVPW (mm) } \\
\text { IVS (mm) }\end{array}$ & $24.80 \pm 5.04$ & $23.54(11.25-34.27)$ \\
\hline
\end{tabular}

\section{Results}

We calculated the mean diurnal and nocturnal values of SBP and DBP from the 24-hour ambBP profile and compared them with the BP percentiles, according to the Task Force on Blood Pressure Control in Children [9].

No patient presented mean diurnal SBP or DBP suggestive of hypertension (defined as BP levels superior to 95th percentile). No patient presented mean SBP or DBP in the high normal BP range (defined as BP between 90th and 95th percentile). Mean diurnal SBP and DBP were significantly higher than nocturnal BP levels (diurnal SBP: $107.29 \mathrm{mmHg}$ \pm 7.14 , nocturnal SBP: $97.43 \mathrm{mmHg} \pm 7.43-P:<.0001$; diurnal DBP: $62.57 \mathrm{mmHg} \pm 5.08$, nocturnal DBP: 53.33 $\mathrm{mmHg} \pm 4.44-P:<.0001)$. At maximal heart rate during Bruce test, SBP was in the normal range in all patients, while 7 patients $(33,3 \%-6 \mathrm{~F}, 1 \mathrm{M})$ presented high DBP (between the 90th and the 95th centile). The results of ambBP profile and excBP are showed in Table 2.

We did not find any gender related differences in 24hour ambBP profile, excBP and myocardial hypertrophy parameters.

Multivariable linear regression showed that mean diurnal SBP, from the 24-hour ambBP profile, was negatively related to $17-\mathrm{OH}$-progesterone levels $(P$ : .026, 95\% CI: -0.34 to $-0.02, \beta=-0.18)$, while mean diurnal DBP was positively related to age ( $P$ : .003, 95\% CI: 0.29 to $1.28, \beta=0.79)$ and to height SDS ( $P$ : .029, 95\% CI: 0.22 to $3.52, \beta=1.87)$. Mean nocturnal DBP was affected by testosterone levels $(P: .016$, 95\% CI: 0.002 to $0.021, \beta=0.01)$. SBP at maximal heart rate during exercise was influenced by age $(P<.0001,95 \% \mathrm{CI}$ : 


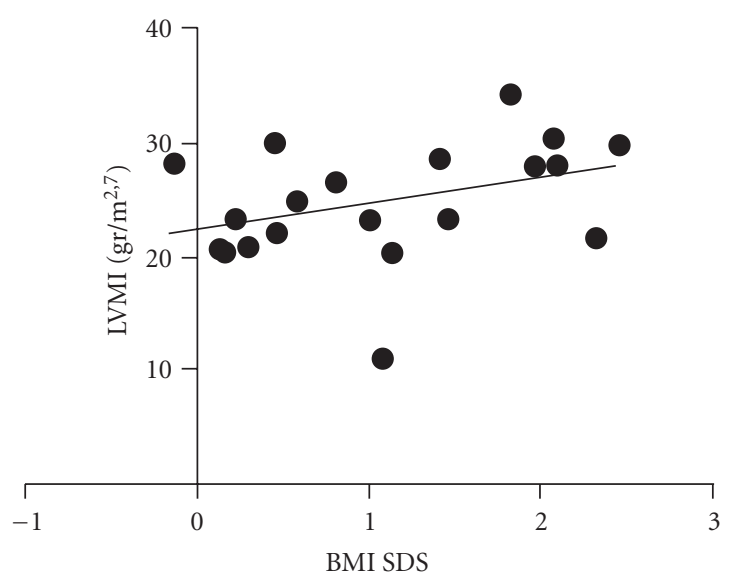

FIgURE 1: Correlation between BMI SDS and LVMI ( $P$ : 0.044).

1.79 to $4.67, \beta=3.23)$, whereas DBP showed a correlation both with age $(P:<.0001,95 \%$ CI: 1.30 to $3.03, \beta=2.20)$ and with BMI SDS ( $P$ : .033 , 95\% CI: 0.40 to $8.76, \beta=4.58$ ). No $\mathrm{CAH}$ patient presented left ventricular hypertrophy, defined by the published paediatric criteria (LVMI > 38.6 g/m $\mathrm{m}^{2.7}$ ). Multivariable linear regression showed that LVM was affected by height SDS ( $P$ : .007, 95\% CI: 2.67 to $14.17, \beta=8.42$ ), by age $(P:<.0001,95 \% \mathrm{CI}: 2.12$ to $5.82, \beta=3.87)$ and by testosterone levels ( $P$ : .008, 95\% CI: 0.01 to $0.09, \beta=0.053)$. LVMI was positively related to BMI SDS ( $P$ : .044, 95\% CI: 0.09 to $6.17, \beta=3.13$-Figure 1 ), and to testosterone levels ( $P$ : .031, 95\% CI: 0.002 to $0.035, \beta=0.018)$. LVPW was affected only by age ( $P: .008,95 \%$ CI: 0.03 to $0.19, \beta=0.11)$.

$\mathrm{HC}$ dose did not influence 24-hour ambBP profile, excBP, and echocardiographic parameters.

\section{Discussion}

Our data suggest that ambBP profile and excBP are substantially normal in young $\mathrm{CAH}$ patients with good hormonal balance. Our CAH patients were not over treated, despite a fairly good hormonal balance. Mean HC dose corresponded nearly to a replacement dose and it could not be considered as supra physiologic. In this conditions nocturnal systolic and diastolic BP dips were maintained; neither ambBP profile nor excBP were affected by $\mathrm{HC}$ dose. A few recent studies, investigating $\mathrm{BP}$ profile in $\mathrm{CAH}$ patients, have reported conflicting results.

Roche et al. [15] in 2003 studied with ambBP monitoring 37 children, aged between 6.1 and 18.2 years, affected by SW CAH. Mean daytime SBP was higher than the reference population, $58 \%$ of patients had systolic hypertension. Mean daytime DBP was also elevated and $24 \%$ patients had diastolic hypertension. In $84 \%$ of patients the physiological nocturnal dip of SBP was absent. They demonstrated that higher BP levels in $\mathrm{CAH}$ patients are positively correlated with higher BMI. De Silva et al. [16] in 2004 showed a normal 24-hour ambBP profile in $11 \mathrm{CAH}$ patients. All patients were on GCs replacement therapy at supra-physiologic doses. Mean SBP and DBP during awake and sleep period, systolic and diastolic BP loads and dips were calculated from the ambBP and compared to Task Force References for BP. None of the patients showed BP loads above the 95th percentile.

As previously described in healthy subjects, in our $\mathrm{CAH}$ patients, BP seems to be primarily related to age, BMI and height. In 2006 Völkl et al. [17] analysed the effect of BMI, fat mass (skin fold thickness), GCs and MCs treatment and skeletal maturation on BP. They studied 55 Caucasian classical CAH subjects (45 SW and $10 \mathrm{SV} ; 32$ females and 23 males), aged between 5 and 19 years. All of them were treated with GCs, 53 received additional MCs. A BMI higher than +2 SDS was found in $27.3 \%$ of patients, versus $2.27 \%$ of the general population. Mean diurnal and nocturnal SBP levels were significantly elevated. BMI and serum leptin were positively correlated with skin fold thickness. No correlation between GCs dose and BP was found. The authors concluded that obese CAH children and adolescents presented an higher risk to develop systolic hypertension.

The same group [18] analysed BMI SDS in children and adolescents affected by CAH. A BMI greater than +2 SDS was found in $16.8 \%$ of CAH patients. The BMI SDS did not differ between children treated by different GCs (HC, prednisone or dexamethasone), but it was significantly correlated to the equivalent HC dose. Knorr and Hinrichsende-Lienau [19] found a significant relationship between over treatment with $\mathrm{HC}$ during the first 2 years of age and later development of obesity among young adults. Our $\mathrm{CAH}$ patients showed a mean BMI SDS in the normal range, with only one obese subject (BMI: $32 \mathrm{~kg} / \mathrm{m}^{2}$ ). Nevertheless, DBP at maximal heart rate during exercise and LVMI showed a significant positive correlation with BMI SDS, confirming that these parameters can be considered as early warnings for hypertension and cardiac overload in obese patients.

In our study group, mean diurnal SBP was negatively correlated to 17-OH-progesterone levels, suggesting that a poor hormonal balance, with mild GCs and MCs deficiency and high levels of androgen precursors, could determine blood hypotension. Conversely, mean nocturnal DBP was positively correlated to testosterone levels, suggesting that higher nocturnal DBP could represent and early effect of androgen excess. We did not find any gender related differences in 24-hour ambBP, excBP, and myocardial hypertrophy parameters. The lack of gender dimorphism in cardiovascular parameters could be related to the lower differences in androgen levels between males and females in young $\mathrm{CAH}$ patients.

Myocardial hypertrophy with increased left ventricular wall thickness and greater LVMI had been repeatedly described in subjects with high GCs levels due to Cushing's syndrome $[20,21]$.

Androgenic anabolic steroids, used for improving physical performance, have been considered responsible for myocardial hypertrophy, acute myocardial infarction and sudden death in humans [22] and animal models [23].

On the contrary, left ventricular hypertrophy and its correlation with androgen levels and GCs therapy had never been investigated in $\mathrm{CAH}$ patients. We did not find any evidence of myocardial hypertrophy (LVMI > 38.6 g/m $\mathrm{m}^{2.7}$ ) in our study group. A normal mean LVMI confirms that the 
mean HC dose usually used is not really supra physiologic, in spite of a good hormonal balance. On the other hand, testosterone levels positively affect myocardial hypertrophy parameters (LVM and LVMI), suggesting that androgens excess could have a detrimental effect on myocardial function. Due to the small sample size, these data should not be considered as conclusive. Particularly in the 2 pubertal males, we cannot exclude a normal influence of increasing gonadal androgens on LVM and LVMI.

\section{Conclusions}

The present study suggests that BP levels are substantially normal in young CAH patients. BP levels are not influenced by $\mathrm{HC}$ dose, at least when the doses are not straightforwardly supra physiologic. As described in healthy subjects, age, height and BMI are the main determinants of BP; high DBP during exercise and LVMI indicate an early warning sign for hypertension, positively related to BMI. High androgen levels in CAH patients seem to be an additional long term risk factor for hypertension and myocardial hypertrophy. The small sample size and the wide age span are significant limitations of our study and do not allow a generalization of the results.

If confirmed in larger trials, the increasing of mean nocturnal DBP, LVM and LVMI could represent early markers of cardiovascular damage due to androgen excess. The systematic monitoring of BP, BMI, and eventually echocardiography is important to recognize early changes of these parameters and to prevent cardiovascular morbidity in adult $\mathrm{CAH}$ patients.

\section{Acknowledgment}

The authors greatly appreciate the secretarial assistance of Mrs. Luciana Luciani.

\section{References}

[1] P. C. Hindmarsh, "Management of the child with congenital adrenal hyperplasia," Best Practice and Research, vol. 23, no. 2, pp. 193-208, 2009.

[2] W. Chemaitilly, R. C. Wilson, and M. I. New, "Hypertension and adrenal disorders," Current Hypertension Reports, vol. 5, no. 6, pp. 498-504, 2003.

[3] D. P. Merke, G. P. Chrousos, G. Eisenhofer, et al., "Adrenomedullary dysplasia and hypofunction in patients with classic 21-hydroxylase deficiency," The New England Journal of Medicine, vol. 343, no. 19, pp. 1362-1368, 2000.

[4] E. Charmandari, G. Eisenhofer, S. L. Mehlinger, et al., "Adrenomedullary function may predict phenotype and genotype in classic 21-hydroxylase deficiency," The Journal of Clinical Endocrinology \& Metabolism, vol. 87, no. 7, pp. 30313037, 2002.

[5] K. Rahmouni, M. L. G. Correia, W. G. Haynes, and A. L. Mark, "Obesity-associated hypertension: new insights into mechanisms," Hypertension, vol. 45, no. 1, pp. 9-14, 2005.

[6] G. Bjelaković, S. Beninati, D. Pavlović, et al., "Glucocorticoids and oxidative stress," Journal of Basic and Clinical Physiology and Pharmacology, vol. 18, no. 2, pp. 115-127, 2007.
[7] J. L. Arriza, C. Weinberger, G. Cerelli, et al., "Cloning of human mineralocorticoid receptor complementary DNA: structural and functional kinship with the glucocorticoid receptor," Science, vol. 237, no. 4812, pp. 268-275, 1987.

[8] E. Charmandari, M. Weise, S. R. Bornstein, et al., "Children with classic congenital adrenal hyperplasia have elevated serum leptin concentrations and insulin resistance: potential clinical implications," The Journal of Clinical Endocrinology \& Metabolism, vol. 87, no. 5, pp. 2114-2120, 2002.

[9] E. J. Roccella, "Update on the 1987 Task Force Report on High Blood Pressure in Children and Adolescents: a working group report from the National High Blood Pressure Education Program Working Group on Hypertension Control in Children and Adolescents," Pediatrics, vol. 98, no. 4, part 1, pp. 649-658, 1996.

[10] J. D. Kay, A. R. Sinaiko, and S. R. Daniels, "Pediatric hypertension," American Heart Journal, vol. 142, no. 3, pp. 422-432, 2001.

[11] M. Cappa, C. Bizzarri, C. Martinez, et al., "Neuroregulation of growth hormone during exercise in children," International Journal of Sports Medicine, vol. 21, no. 2, pp. S125-S128, 2000.

[12] D. J. Sahn, A. DeMaria, J. Kisslo, and A. Weyman, "Recommendations regarding quantitation in M-mode echocardiography: results of a survey of echocardiographic measurements," Circulation, vol. 58, no. 6, pp. 1072-1083, 1978.

[13] C. Hanevold, J. Waller, S. Daniels, R. Portman, and J. Sorof, "The effects of obesity, gender, and ethnic group on left ventricular hypertrophy and geometry in hypertensive children: a collaborative study of the International Pediatric Hypertension Association," Pediatrics, vol. 113, no. 2, pp. 328333, 2004.

[14] S. R. Daniels, "Hypertension-induced cardiac damage in children and adolescents," Blood Pressure Monitoring, vol. 4, no. 3-4, pp. 165-170, 1999.

[15] E. F. Roche, E. Charmandari, M. T. Dattani, and P. C. Hindmarsh, "Blood pressure in children and adolescents with congenital adrenal hyperplasia (21-hydroxylase deficiency): a preliminary report," Clinical Endocrinology, vol. 58, no. 5, pp. 589-596, 2003.

[16] K. S. H. de Silva, S. Kanumakala, J. J. Brown, C. L. Jones, and G. L. Warne, "24-hour ambulatory blood presurre profile in patients with congenital adrenal hyperplasia-a preliminary report," Journal of Pediatric Endocrinology and Metabolism, vol. 17, no. 8, pp. 1089-1095, 2004.

[17] T. M. K. Völkl, D. Simm, J. Dötsch, W. Rascher, and H. G. Dörr, "Altered 24-hour blood pressure profiles in children and adolescents with classical congenital adrenal hyperplasia due to 21-hydroxylase deficiency," The Journal of Clinical Endocrinology \& Metabolism, vol. 91, no. 12, pp. 4888-4895, 2006.

[18] T. M. K. Völkl, D. Simm, C. Beier, and H. G. Dörr, "Obesity among children and adolescents with classic congenital adrenal hyperplasia due to 21-hydroxylase deficiency," Pediatrics, vol. 117, no. 1, pp. e98-e105, 2006.

[19] D. Knorr and S. G. C. Hinrichsen de Lienau, "Persistent obesity and short final height after corticoid overtreatment for congenital adrenal hyperplasia (CAH) in infancy," Acta Paediatrica Japonica, vol. 30, supplement, pp. 89-92, 1988.

[20] M. L. Muiesan, M. Lupia, M. Salvetti, et al., "Left ventricular structural andnfunctional characteristics in Cushing's syndrome," Journal of the American College of Cardiology, vol. 41, no. 12, pp. 2275-2279, 2003. 
[21] F. Fallo, P. Maffei, A. Dalla Pozza, et al., "Cardiovascular autonomic function in Cushing's syndrome," Journal of Endocrinological Investigation, vol. 32, no. 1, pp. 41-45, 2009.

[22] S. E. Campbell, A. Farb, and K. T. Weber, "Pathologic remodeling of the myocardium in a weightlifter taking anabolic steroids," Blood Pressure, vol. 2, no. 3, pp. 213-216, 1993.

[23] A. Malhotra, P. Buttrick, and J. Scheuer, "Effects of sex hormones on development of physiological and pathological cardiac hypertrophy in male and female rats," American Journal of Physiology, vol. 259, no. 3, pp. H866-H871, 1990. 